\title{
Impact of clinical interventions in the timely diagnosis and therapeutic approach of necrotizing soft tissue infections
}

\begin{abstract}
This review aims to analyze the role of specific diagnostic and therapeutic interventions with a positive impact on the necrotizing soft tissue infection approach, we underline strategies that - conduct in a timely manner - may prevent patients death, as well as to avoid limb loss. We discuss the usefulness of clinical findings, imaging and biochemical markers in the address of infection. Also, we present an analysis of hyperbaric oxygen therapy in the role of the disease.
\end{abstract}

Keywords: necrotizing soft tissue infections, gas gangrene, hyperbaric oxygen, necrotizing fascitis, magnetic resonance imaging, clostridial myonecrosis
Volume 4 Issue 4 - 2019

\author{
Adrián Fallas Mora,' Cristian Gomez \\ Murillo, ${ }^{2}$ Grettel Rojas Soto, ${ }^{2}$ Carlos Andrey \\ Zumbado Salazar ${ }^{3}$ \\ 'Microbology Department, Microbiologist and Clinical \\ Chemistry, University of Costa Rica, Costa Rica \\ ${ }^{2}$ Microbiology Department, Microbiologist and Clinical \\ Chemistry, San Francisco de Asís Hospital, Costa Rica \\ ${ }^{3} \mathrm{Head}$ of Microbiology Department, Microbiologist and Clinical \\ Chemistry, San Francisco de Asís Hospital, Costa Rica
}

\begin{abstract}
Correspondence: Adrian Fallas Mora, Department of Microbiology, Microbiologist and Clinical Chemistry, San Francisco de Asís Hospital and student of the Specialty Postgraduate in Medical Bacteriology, University of Costa Rica, Costa Rica,Tel+50687760 I I I, Email adrifallasm@gmail.com
\end{abstract}

Received: July 03, 2019 | Published: July 08, 2019

\section{Introduction}

Necrotizing soft tissue infections (NSTIs) are a group of clinical entities characterized by progressive and fulminant destruction of rapidly evolving tissues, associated to high mortality rates due to systemic toxicity and multiorgan failure. ${ }^{1}$ The microbiological classification of NSTIs by subtypes is very useful to review therapeutic and diagnostic schemes. Type 1 is the most prevalent, comprising polymicrobial infections by aerobic and anaerobic bacteria. The predominant isolates are species of the Streptococcus, Staphylococcus, Enterococcus, Pseudomonas, Acinetobacter, Bacteriodes generas and members of the Enterobacteriacea family. Clostridium commonly participates in this context without causing myonecrosis. Events that compromise peripheral vascularization are the classic risk factor for acquiring the infection, where interestingly, no triggering events where identified in $20-50 \%$ of patients. ${ }^{2}$ The Fournier Gangrene, diabetic foot infection and necrotizing fasciitis are classic infections of this group. It also includes complications of odontogenic infections caused by Fusobaterium, Peptostreptococcus, Bacteriodes, anaerobic Streptococcus and spirochetes..$^{1-2}$ Type 2 NSTIs are monomicrobial caused by Streptococcus group A, some are accompanied by community-acquired methicillin-resistant Staphylococcus aureus (CA-MRSA). Unlike the first group, these infections can occur in patients without predisposing factors. ${ }^{1-2}$

Finally, Type 3 NSTIs includes monomicrobial infections attributed to Clostridium, Vibrio vulnificus or Aeromonas sp. The first one have a strong association to trauma and surgeries. With the improvement in surgical interventions, the current epidemiology has changed to injuries in patients with intravenous (IV) drug abuse.
Clostridium perfringens is the etiologic agent in $70-80 \%$ of cases. The remaining genera are associated with similar infectious processes. The exhaustive approach in clinical history is necessary to establish differential diagnosis, specifically to investigate contact with different water sources. ${ }^{1-2}$

The key to the timely therapeutic approach of NSTIs is the early diagnosis. It is imperative to recognize them early since delays in treatment have a high association with extensive tissue destruction and consequent limb loss ${ }^{1-2}$ However, the clinical presentation is not clear; there is usually absence of classic signs and symptoms. The treatment strategy includes antimicrobial therapy and aggressive surgical debridement. ${ }^{1-3}$ There is a flow of information on complementary techniques to this basic therapy, such as the therapeutic strategy of hyperbaric oxygen (HBO), blood infusions and intravenous immunoglobulins. In turn, there are multiple published clinical guidelines for comprehensive management, however; its application is limited. ${ }^{2}$ This review aims to analyze the role of specific diagnostic and therapeutic interventions with positive impact on the NSTIs approach, on time to prevent patient deaths, as well as to avoid limb loss.

\section{Discussion}

Despite the difficulties presented by the patient's exploration, this process is crucial to establish an opportune diagnosis. It is important to note that the positive predictive value (PPV) of pathognomonic physical findings isn't surpassed by any other technology. ${ }^{1-4}$ The localized pain is perhaps the symptom of first appearance; it can be accompanied by erythema, hyperthermia, edema around the erythematous zone, epidermolysis, tachycardia, fever and tanning 
of the skin. Late physical findings include hemorrhagic bullae, bad odor, dermal gangrene, crepitus, seropurulent drainage, inflammatory response syndrome and progression of early symptoms. The disproportionate pain of sudden onset is highly suggestive of NSTIs, unfortunately, at this level the tissue involvement is already large and irreversible, with imminent amputation of the affected limb in most cases. ${ }^{2-4}$ Tissue involvement in the same way is variable, ranging from subcutaneous necrosis to sepsis with injuries in fascia and muscles. Although, it methods for early detection from serological markers have been proposed. The laboratory risk indicator for necrotizing fasciitis (LRINEC) is a strategy that considers C-reactive protein, white blood cell count, hemoglobin concentration, sodium, creatinine and serum glucose to establish a risk score. ${ }^{5}$ The above parameters are integrated in a risk indicator of necrotizing fasciitis. ${ }^{5}$ This score has had limited acceptance by the medical community, which suffers from the lack of correlation with reality, where leukocytosis and hyponatremia do not occur simultaneously. It is important to note that this tool was developed exclusively with laboratory parameters ignoring physical findings; in this sense, it is difficult to reproduce results in the scenario of a disease with such variability in its clinical presentation. In addition, when there is a high suspicion of necrotizing fasciitis throughout the history, physical examination, LRINEC should not be calculated. The clinician must go directly to surgical debridement. In summary, the approach with biochemical scores has utility in the exclusion of NSTIs, but not in its identification. A high index of clinical suspicion remains the paramount. ${ }^{5}$

The scope of microbiological studies in early diagnosis of NSTIs is limited. Fluid aspiration for detection of microorganisms in initial stages has low VPP. ${ }^{1}$ Additionally, the presence of bacteria does not always correlate with the infectious process from this sample. In fact, absence of drain does not rule out tissue commitment either. Gram staining can be definitive in the diagnosis of some NSTIs. However, the sample that presents adequate sensitivity is the tissue obtained during surgical exploration. ${ }^{1-2}$ Therefore, this intervention does not contribute to early diagnosis. Cultures take a long time to complete, so that few authors consider them in algorithms. ${ }^{1-3}$ Therefore, microbiological techniques inclusion in diagnostic algorithms are interventions without any clinical impact.

In patients with physical findings that do not support urgent debridement, imaging techniques can clarify tissue involvement and define whether the clinical picture corresponds to NSTIs. ${ }^{6}$ However, it should not be forgotten that in presence of clear signs and symptoms, surgical intervention without any delay of images is mandatory. NSTIs are characterized by massive tissue destruction; the initial presentation can be restricted to deep planes, preserving intact skin..$^{2-5}$

It is important to consider the abscense of universally accepted imaging protocol to address patients with suspected NSTIs. ${ }^{6}$ Techniques such as computed tomography (CT), flat X-rays and magnetic resonance imaging (MRI) have been used, but none of those is the gold standard..$^{6,7}$

The use of images seeks to demonstrate pathognomonic and complementary features to guide the diagnosis of NSTIs. In this sense, flat X-rays are excluded from the scenario, as they rarely show the presence of gas. Previous studies reported the finding in 25-47.7\% of patients. Although this characteristic is of variable appearance, it has high specificity for the diagnosis. Current use of simple X-rays is limited to ruling out underlying bone injuries or presence of foreign bodies. $^{6}$
Previously, CT was questioned because of its low sensitivity and specificity in the diagnosis of necrotizing infections. ${ }^{1}$ However, most studies were based solely on gas detection. This finding has a great impact for the early detection of gas gangrene. Clinical signs and symptoms such as subcutaneous crepitus and disproportionate pain of the extremity added to the presence of gas in subcutaneous tissue is pathognomonic of the Clostridial infection. Therefore, previous reports restricted the use of CT only to address this particular NSTI. ${ }^{1-6}$ Its capacity to guide diagnosis of the spectrum of necrotizing infections has increased significantly with updated technology, introduction of contrast media and updating criteria. ${ }^{7}$ Inflammatory changes observation under fascias, presence of multiple fluid collections and unequal contrast medium distribution has been incorporated ${ }^{7}$ With the inclusion of these variables, high sensitivity and Negative Predictive Value (NPV) are obtained, in one study 100\% was reported for both parameters, suggesting its use to reliably rule out the need for surgical intervention in patients with high suspicion of NSTIs. ${ }^{7}$

A score for NSTIs diagnosis based on CT have been proposed. Gas presence within fascia, muscular edema, fluid collection, lymphadenopathy and subcutaneous edema is contemplated. ${ }^{4}$ The developer research group has shown striking evidence, however the studies carried out were retrospective, including patients without NSTIs as control group, ${ }^{8}$ this approach introduce significant heterogeneity. ${ }^{4}$ In addition to its diagnostic value, CT can delimitate surgical exploration of suspicious areas, especially in presence of extensive edema or involvement of difficult anatomical areas, such as the retroperitoneum and mediastinum. ${ }^{7}$ This is intended to debride all affected areas and avoid further complications. Deep abscesses are approached correctly with $\mathrm{CT}$, contrary to clinical examination where they can go unnoticed. ${ }^{1}$

MRI can accurately differentiate necrotizing infections from nonnecrotizing infections. ${ }^{6}$ Due to its high spatial resolution and inherent contrast, this technique provides accurate information on anatomical compromise, many guidelines consider MRI the best aproach to evaluate anomalies in the musculoskeletal system, including infection. ${ }^{9}$ The findings allow clinicians to confirm or exclude the diagnosis of NSTIs, in addition to clearly establish the distribution and margins of infected areas. With this technique, multiplanar images can be obtained without the use of ionizing radiation, which is an advantage when using TC. ${ }^{9}$

Unfortunately, the time invested taking images for MRI does not justify its use, especially in patients with sepsis who may be subjected to other procedures simultaneously. ${ }^{1-2}$ Additionally, these scans require complete immobilization, where any movement can lead to exhaustive and unproductive interpretations, in a way that would further delay diagnosis. ${ }^{7}$ On the other hand, MRI is not as useful as CT for gas detection in soft tissues. This technique tends to overestimate the involvement of deep tissues; therefore, it cannot be used to reliably distinguish between necrotizing cellulitis and deeper infection. ${ }^{7-9}$

Tissue biopsies provide reliable results in NSTIs. It is based on the observation of histological changes that include tissue necrosis, polymorphonuclear infiltration, vascular thrombosis and sometimes microorganisms within the destroyed tissue. Despite the valuable information provide, clinical experience is limited, since the pathology experts are not available 24 hours a day to perform the interpretation in a timely manner. ${ }^{1-3}$

Once the diagnosis of NSTIs is establish, classic treatment 
can be complemented with alternative therapies, such as exposure to hyperbaric oxygen (HBO). The strategie consists in oxygen administration under pressure exceeding 1 absolute atmosphere, achieving dramatic increase in oxygen tension within the tissues. The benefits are direct bactericidal/bacteriostatic activity, optimization of immune response and additive or synergistic effect with antimicrobials. The main target are anaerobic bacteria; as they have few detoxification mechanisms against reactive oxygen species. ${ }^{10,11}$

Increases in oxygen tension enhances host defense mechanisms, particularly neutrophil bactericidal activity. Although degranulation of polymorphonuclear cells can operate in a hypoxic state, death induction due to oxidative damage is totally dependent on oxygen, which is why the requirement for this element increases up to 15 times during NSTIs. On the other hand, in the pathogenesis of gas gangrene and streptococcal myonecrosis, neutrophil adherence increase to epithelium vascular cells promotes hypoxia establishment and pathogen survival as a consecuence. ${ }^{10,11}$

Regarding antimicrobial action mechanisms, HBO usefullness to enhance antibiotic effect in infections caused by aerobic bacterias has been exhaustively demonstrated. The higher the concentration of dissolved oxygen, the optimal levels for action of aminoglycosides, fluoroquinolones, trimethoprim-sulfamethoxazole, vancomycin and some sulfonamides. However, this potentiation have limited effect against anaerobic bacteria implicated in most NSTIs. ${ }^{11} \mathrm{HBO}$ incorporation in clinical guidelines is restrict to gas gangrene, necrotizing fasciitis, diabetic foot infections, refractory osteomyelitis, neuro surgical infections and some fungal infections. Like MRI, this approach cannot be applied in patients who require multiple interventions at the same time, especially medical emergencies scenarios. Time of transfer to the hyperbaric chamber and delay by pressure compensation should also be considered. ${ }^{10,11} \mathrm{HBO}$ should never be used in monotherapy for NSTIs. ${ }^{1,3,10,11}$ It is indicated in the cases mentioned as complementary therapy, included in clinical guidelines of the Hyperbaric Medical Society and the European Committee of Hyperbaric Medicine. ${ }^{2}$ HBO inhibits toxin synthesis and reproduction of Clostridium well as detoxification of circulating toxins. ${ }^{10}$ In addition, exposure to prolonged oxygen prevents tissue loss and promotes wound healing. The previous findings support HBO therapy for gas gangrene treatment; it is complementary and does not replace surgical debridement. The proposed approach suggests starting with $\mathrm{HBO}$, then entering the operating room to remove necrotic tissue. This sequence has proven to be beneficial, since it easily demarcates the affected area and establishes a better limit for debridement. ${ }^{11}$ The antimicrobial treatment should be initiated as soon as possible. On the other hand, necrotizing fasciitis can be treated with HBO to extend patient's survival, being a complementary therapy to first-order interventions such as surgical and antimicrobial treatment. Subsequent sessions in the hyperbaric chamber are required to avoid re-entry to the operating room and to reduce healing time.${ }^{11} \mathrm{HBO}$ has been also indicated in chronic stages of diabetic foot infections that do not heal with aggressive treatments. Some medical centers prescribe this therapy to patients with wounds Wagner grade 3 or higher, who failed in a standard 30-day scheme based on correction of vascularization, optimization of nutritional status, glucose control, surgical debridement and antimicrobial treatment. ${ }^{10}$ The controversy with HBO lies in the absence of properly conducted clinical trials, with cases and controls that demonstrate clinical efficacy. ${ }^{2}$ The studies published to date only support its use in NSTIs type 1 (polymicrobial).
It is necessary to consider that randomized trials in conditions such as gas gangrene is not possible, since it is not considered ethical to use a control group, as there is theoretical evidence of benefit with the use of HBO in anaerobic infections. Additionally, there is little information that specifies the standardized time of exposure, timely application intervals during infection, days of therapy, among others. Many studies support application protocols until focus control and physiological abnormalities resolution. , $^{10,11}$

Side effects reports during $\mathrm{HBO}$ is unusual and requires minimal treatment. Barotraumas, defined as trauma induced by pressure in the middle ear, are a recognized self-limited complication, they mainly occur in patients patient with assisted mechanical ventilation. The worst scenarios have been reports of acute cerebral toxicity due to oxygen accompanied by seizures. ${ }^{12}$ Blood purification by plasmapheresis is another therapeutic strategy of great value in the NSTIs intervention. It is based on removal of molecular mediators proposed as causal of the NSTIs pathophysiology and its consequent sepsis. $^{2}$ Deep hypoalbuminemia is also common, therefore, substitution with colloid (albumin) may be necessary to maintain oncotic pressure. Red blood cell transfusions may be needed to treat extravascular hemolysis generated by bacterial toxins. Hemolysins cause surprising Hematocrit depletion without disseminated intravascular coagulopathy. Therefore, hematocrit may be a better indicator for red blood cell transfusion than hemoglobin. ${ }^{1-3}$

\section{Conclusions}

The NSTIs continue to present a diagnostic challenge in the daily clinical practice. Current radiological techniques have shown to guide surgical exploration, generating a significant impact on patient survival. Due to its characteristics, CT is the most feasible method to incorporate into diagnostic algorithms. The use of HBO has been widely explored as an adjunct to conventional therapy; however, clinical data only support its use in type 1 NSTIs. These and other strategies can be incorporated in the approach of necrotizing infections, being clear their role as a complement to the basic action plan, which includes diagnosis based on exhaustive physical examination, antimicrobial administration and surgical debridement. The early application of these measures is crucial to ensure patient survival.

\section{Acknowledgments}

None.

\section{Conflicts of interests}

The authors does not declare any conflicts of interest.

\section{References}

1. Cocanour CS, Chang P, Huston JM, et al. Management and Novel Adjuncts of Necrotizing Soft Tissue Infections. Surg Infect. 2017;18(3):250-272.

2. Stevens DL, Bisno AL, Chambers HF, et al. Practice Guidelines for the Diagnosis and Management of Skin and Soft Tissue Infections: 2014 Update by the Infectious Diseases Society of America. Clin Infect Dis. 2014;59(2):10-52.

3. Kamath RS, Sudhakar D, Gardner JG, et al. Guidelines vs Actual Management of Skin and Soft Tissue Infections in the Emergency Department. Open Forum Infect Dis. 2018;5(1):188. 
4. Henry SM, Davis KA, Morrison JJ, et al. Can necrotizing soft tissue infection be reliably diagnosed in the emergency department ? Trauma Surg Acute Care Open. 2018;3(1):157.

5. El Menyar A, Asim M, Mudali IN, et al. The laboratory risk indicator for necrotizing fasciitis (LRINEC) scoring: the diagnostic and potential prognostic role. Scand J Trauma Resusc Emerg Med. 2017;25(1):28.

6. Leichtle SW, Tung L, Khan M, et al. The role of radiologic evaluation in necrotizing soft tissue infections. J Trauma Acute Care Surg. 2016;81(5):921-924.

7. Martinez M, Peponis T, Hage A, et al. The Role of Computed Tomography in the Diagnosis of Necrotizing Soft Tissue Infections. World J Surgery. 2018;42(1):82-87.

8. McGillicuddy EA, Lischuk AW, Schuster KM, et al. Development of a Computed Tomography-Based Scoring System for Necrotizing SoftTissue Infections. J Trauma. 2011;70(4):894-899.

9. Soldatos T, Durand DJ, Subhawong TK, et al. Magnetic Resonance Imaging of Musculoskeletal Infections. Acad Radiol. 2012;19(11):14341443.
10. AL Gill, CNA Bell. Hyperbaric oxygen: its uses, mechanisms of action and outcomes. QJM. 2004;97(7):385-395.

11. Shaw JJ, Psoinos C, Emhoff TA, et al. Not just full of hot air: hyperbaric oxygen therapy increases survival in cases of necrotizing soft tissue infections. Surg Infect. 2014;15(3):328-335.

12. Heyboer M, Sharma D, Santiago W, et al. Hyperbaric Oxygen Therapy: Side Effects Defined and Quantified. Adv Wound Care. 2017;6(6):210 224. 